\title{
Differences in Newborn Screening Results Among Women with Gestational Diabetes Mellitus
}

Journal of Inborn Errors of Metabolism \& Screening 2021, Volume 9: e20200013 DOI: https://doi.org/10.1590/2326-4594JIEMS-2020-0013

\author{
Jane Estrella ${ }^{\oplus}$, Veronica Wiley ${ }^{2,3}$, Jincy Immanuel ${ }^{4}$ \\ and David Simmons ${ }^{5}$ (B)
}

\begin{abstract}
Multiple studies undertaken on cord blood demonstrate analyte perturbations in infants exposed to gestational diabetes mellitus (GDM). Cord blood as a sample is influenced by maternal and placental metabolism. Newborn screening (NBS), performed after the first 24 hours of life reflects early neonatal metabolism. We compared NBS analytes between women with and without GDM with different management approaches in the Treatment of Booking of Gestational Diabetes (TOBOGM) pilot randomised controlled trial. Pregnant women with GDM risk factors were randomised to early or deferred GDM treatment following an oral glucose tolerance test (<20 weeks gestation). Women without GDM served as "decoys". From the decoy group 11 developed GDM (screened at 26-28 weeks), were analysed separately; their results were compared with the other groups. De-identified controls were chosen from NBS results from the same analytic run matched for sex, birthweight and gestational age. Results were available for 73/78 women participating in the pilot and 358 de-identified controls. Tyrosine levels ( $\mu \mathrm{mol} / \mathrm{l}$; whole blood)were higher in the late GDM group vs early, deferred treatment, and decoy groups (medians:106.28; IQR: 96.73-151.11) (76.33; 64.64-97.90) (75.68; 66.59-110.88)(73.74; 58.32-90.36) $(\mathrm{p}=0.009)$ and remained elevated when compared to normal, age-matched controls (106.28; 96.73-151.11) (87.26; 68.55-111.26) ( $\mathrm{p}$ value=0.01) Immunoreactive trypsinogen ( $\mu g m / l ;$ whole blood)was highest in the early treatment group when compared with group-specific controls $(22.30 ; 13.90-29.90$ vs $14.00,10.60-21.10)(p=0.02)$. These results provide evidence of biochemical perturbations detectable on NBS of in-utero exposure to hyperglycemia and treatment and provide data for hypothesis building.
\end{abstract}

Keywords: Gestational diabetes mellitus, Diabetes in pregnancy, Newborn screening, tyrosine, immunoreactive trypsinogen, IRT.

\section{Introduction}

Newborn screening (NBS) is a successful global public health initiative that has improved outcomes in infants affected by rare disorders, primarily of metabolism, by screening for such disorders soon after birth. The timing of NBS collection (at least 24 hours following birth via a heel-prick) approximates infant metabolism less influenced by the placenta than cord blood and is reflective of neonatal catabolism particularly between periods of feeding, ideal for diagnosis of disorders of fatty oxidation. The research potential in the field of NBS has slowly gained interest, with literature demonstrating the feasibility of using NBS results in gestational age dating, risk prediction for neonatal disorders, and establishing specific acylcarnitine profiles in large for gestational age infants [1-7].

Studies utilizing NBS results in diabetes have been limited to exploring associations between analytes and the development of type 1 and monogenic diabetes [8-14]. Multiple large-scale trials have shown differences in metabolic pathways using untargeted metabolomic techniques from cord blood collected at delivery in infants of women with diabetes in pregnancy

\footnotetext{
${ }^{1}$ Western Sydney University, Macarthur Clinical School, School of Medicine, Sydney, Australia.

${ }^{2}$ NSW Newborn Screening Programme, Sydney, Australia.

${ }^{3}$ University of Sydney, Discipline of Paediatrics and Child Health, Sydney, Australia.

${ }^{4}$ Western Sydney University, Sydney, Australia.

${ }^{5}$ Western Sydney University, Macarthur Clinical School, Sydney, Australia.
}

Received August 22, 2020, and in revised form March 16, 2021. Accepted for publication March 29, 2021.

\section{Corresponding Author:}

David Simmons, Western Sydney University, Macarthur Clinical School, Sydney, Australia.E-mail: da.simmons@westernsydney.edu.au 
$[15,16]$. GDM is usually screened for at the end of the second/ beginning of the third trimester (24-28 weeks), although certain high-risk groups are screened early in pregnancy seeking undiagnosed type 2 diabetes or early GDM [17]. Hyperglycemia in pregnancy represents a spectrum that may predate GDM screening, with glucose levels below diagnostic criteria for overt diabetes in pregnancy [18]. How this should be treated is unclear. The Treatment of Booking Gestational diabetes Mellitus (TOBOGM) pilot randomised controlled trial was conducted to determine the feasibility of a study comparing pregnancy outcomes between women in early pregnancy $(<20$ weeks) with immediate or deferred treatment for their relative hyperglycaemia [19]. TOBOGM therefore creates two groups of infants of women with early GDM, those whose mothers underwent early treatment, and those who were not treated until later ie experienced longer exposure to hyperglycemia in utero. We sought to determine if early treatment of GDM $(<20$ weeks) in the TOBOGM pilot would have any effect on early neonatal metabolism (using NBS as an alternative to cord blood metabolomics) as compared to those who started treatment at 26 weeks onward (standard treatment), and in comparison with women who had risk factors for GDM. As TOBOGM is primarily a randomized-control trial to determine the effects of early treatment (primarily through diet and caloric restriction) of mothers with GDM, we also sought to compare the NBS results of the babies of the early treatment group against those of normal, age-matched controls.

\section{Materials and Methods}

\section{TOBOGM Pilot}

Women with GDM risk factors were screened for GDM at the first antenatal visit ( $<20$ weeks) and were randomized to either early ( $<20$ weeks gestation) or deferred treatment (24-28 weeks). GDM was diagnosed throughout using the International Association of Diabetes in Pregnancy Study Groups criteria using a $75 \mathrm{~g}$ oral glucose tolerance test (fasting $\geq 5.1 \mathrm{mmol} / 11$ hour; $\geq 10.0 \mathrm{mmol} / 1$ 2hour; $\geq 8.5 \mathrm{mmol} / \mathrm{l}$ ) [17]. No women had overt diabetes in pregnancy. Women with GDM diagnosed both early and at 24-28 weeks were treated according to the ADIPS guidelines (diet, physical activity and pharmacotherapy if hyperglycaemia persisted or developed). Women screened with risk factors but no early GDM served as "decoys" so that the women with deferred treatment remained masked.

The control groups (New South Wales Newborn Screening Programme; NSWNSP controls) were de-identified controls chosen from the NBS database (but who were not part of the TOBOGM cohort) using a pragmatic ratio of 1:5 controls for samples analysed immediately before or after the samples of interest ie had the same work date, batch run and hospital of birth, as well as birth weight, sex, and gestational age as much as possible to minimize analytical measurements of uncertainty. Other available data regarding controls were not used for matching (eg feed type, age of the baby at time of collection, time from collection to receipt) Wherever possible, maternal information was matched within the TOBOGM groups, however, we were not able to match all potential maternal confounders, such age, BMI and ethnicity in selecting controls. Data concerning labour, delivery and ethnicity were not available on the NSWNSP samples. Ethics approval was provided by the South Western Sydney Local Health District (SWSLHD) Ethics Committee (Reference number 15/LPOOL/14). Written consent was obtained from all participants prior to inclusion in the study.

\section{Newborn Screening}

Newborn screening results of infants born to mothers who participated in the pilot program were requested from the NSWNSP. Infant results were grouped by maternal study limb.

The majority of NBS were obtained via heel-prick between 48-72 hours after delivery as part of routine clinical care. Blood spots (whole blood) were blotted into NBS collection paper, air-dried and mailed to NSWNSP. Conditions screened in Australia are primary congenital hypothyroidism, cystic fibrosis, galactosemia and over 40 inborn errors of metabolism of amino acids, organic acids and fatty acids [20]. The analyte panel includes thyrotropin (TSH), immunoreactive trypsinogen (IRT), galactose as well as six amino acids, free carnitine, 17 acyl-carnitines and 21 analyte ratios indicative of an inborn error of metabolism. All filter paper cards are analysed on day of receipt when infants age is $<9$ days post birth; after use, all cards are stored in accordance with Ministry of Health policy and discarded after 18 years [21].

\section{Statistical Analysis}

Newborn screening analytes are not normally distributed and therefore reported as medians (interquartile range) rather than mean [22]. Analysis was undertaken using SPSS Version 26.0 (IBM). All matched controls were then grouped and analysed. Differences across the four groups were compared using the Kruskal-Wallis test. Bonferroni correction for pairwise comparisons were performed.

Comparisons of early treatment, deferred treatment and decoy group against their respective control group were undertaken. The decoy group in this trial is representative of normal pregnancies in the presence of risk factors, such as obesity, and a subset were diagnosed with GDM at 24-28 weeks. The results of this group were analysed separately from the rest of the decoy group.

\section{Results}

\section{Demographics}

Seventy-six women participated in the pilot study, all of whom were screened for GDM at the first antenatal visit and grouped into: early treatment i.e. tested and treated for GDM at the first 
antenatal visit (treated group n:11), deferred treatment i.e. tested positive for GDM at booking but deferring treatment until repeat OGTT at 24-28 weeks (no treatment n:9), and women without diabetes at booking (decoys n: 56). Eleven women in the decoy group were diagnosed with GDM following repeat OGTT $(11 / 56 ; 20 \%)$. Figure 1 shows the numbers in each group including NBS controls.

The majority of the women were treated with diet and exercise, with glucose levels titrated to treatment targets (fasting glucose levels $<5.3 \mathrm{mmol} / \mathrm{L}$ and post-prandial glucose levels $<6.8 \mathrm{mmol} / \mathrm{L}$ ). Metformin and/or insulin was added if targets were not met (11/34 women total diagnosed across all groups).
Metformin is known to cross the placenta (in contrast to insulin) but only 3 women in the pilot were treated with metformin.

From the 76, 73 NBS results were matched. One participant had a stillbirth; this sample was excluded. Two NBS results were unavailable (samples unsuitable and required recollection). Sex distribution was roughly equal in the treatment groups, while there was a higher percentage of females $(32 / 53 ; 60 \%)$ born to women in the decoy group.

As a group, infants of mothers with early treatment for early GDM were more likely to be born with a lower birthweight (Table 1). Four infants who were small for gestational age from the early treatment group and 8 infants from the decoy group were admitted to the neonatal intensive care unit.

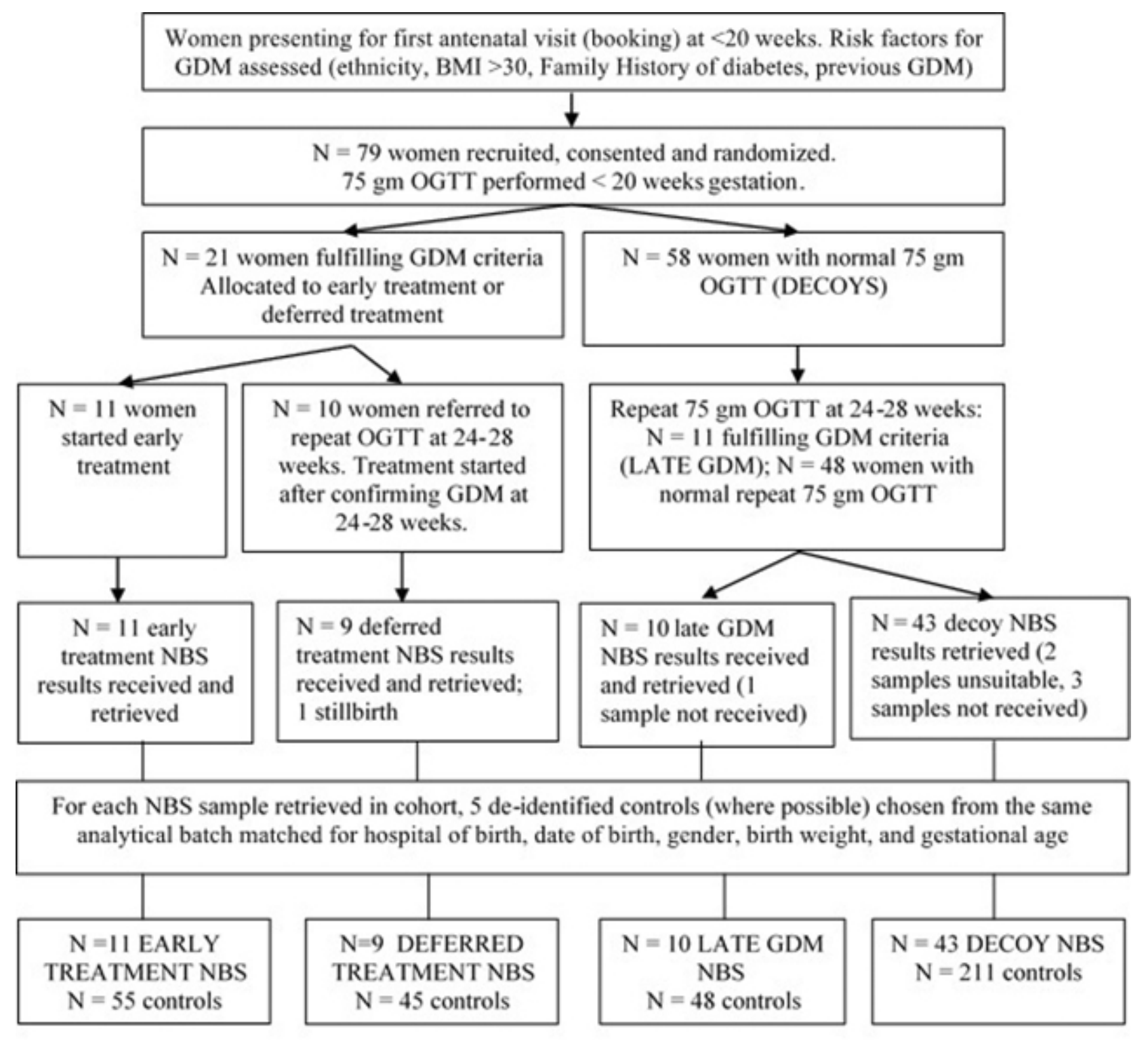

Figure 1. Overview of study groups. GDM=Gestational diabetes mellitus; BMI=Body mass index; OGTT=Oral glucose tolerance test; NBS=Newborn screening. 
Table 1. Comparison of neonatal characteristics.

\begin{tabular}{|c|c|c|c|c|c|}
\hline Characteristics & $\begin{array}{c}\text { Early GDM } \\
\text { Immediate } \\
\text { treatment } \\
\mathbf{N}=11\end{array}$ & $\begin{array}{c}\text { Early GDM } \\
\text { Deferred treatment } \\
\mathbf{N}=9\end{array}$ & $\begin{array}{l}\text { Late GDM } \\
\qquad N=10\end{array}$ & $\begin{array}{c}\text { Decoys } \\
N=43\end{array}$ & $P$ value \\
\hline \multicolumn{6}{|l|}{ Gender (n) } \\
\hline Female & 5 & 4 & 7 & 25 & \\
\hline Male & 6 & 5 & 3 & 18 & 0.60 \\
\hline Birth weight (kg) & $3.06 \pm 0.76$ & $3.64 \pm 0.73$ & $3.28 \pm 0.68$ & $3.36 \pm 0.69$ & 0.33 \\
\hline Gestational age (days) & $268( \pm 10 ; 245-280)$ & $274( \pm 6 ; 266-280)$ & $261( \pm 16 ; 224-273)$ & $270( \pm 15.7 ;$ 196-287)) & 0.28 \\
\hline
\end{tabular}

GDM=Gestational diabetes mellitus; -- missing values. Data are presented as mean士standard deviation, minimum and maximum range. NSWNSP=New South Wales Newborn Screening programme.

\section{Comparison of Early Treatment Group, Deferred Treatment Group, Decoys and Late GDM Group}

Tyrosine was the only analyte $(\mathrm{p}=0.009)$ that was significantly different in the late GDM group from the rest of the cohort (Table 2, Figure 2). This remained statistically significant with Bonferroni correction (early treatment $v s$. late GDM, adj. $\mathrm{p}$ $=0.049$, decoy vs. late GDM, adj. $\mathrm{p}=0.005)$. There was no specific pattern observed in acylcarnitines within the groups (Supplementary Table S1)

\section{Comparison of Early Treatment, Deferred Treatment, Decoy and Late GDM Groups vs NSWNSP Controls}

IRT was higher in the early treated group compared to controls $(p=0.02)$ (Table 3, Figure 3). Tyrosine in the late GDM group was higher compared to their respective controls $(\mathrm{p}=0.01)$. Methionine (MET) was higher in the decoy groups than respective controls $(\mathrm{p}=0.038)$ (Table 3, Figure 4). There were no discernible patterns in the acylcarnitines among the groups (Supplementary Table S1)

Table 2. Analytes of significance across all groups.

\begin{tabular}{lccccc}
\hline Analyte & Early treatment & Deferred treatment & Decoy group & Late GDM & P value \\
\hline Tyrosine $\mu \mathrm{mol} / \mathrm{I}$ & $76.33(64.64-97.90)^{*}$ & $75.68(66.59-110.88)$ & $73.74(58.32-90.36) \dagger$ & $106.29(96.73-151.11)$ & 0.009 \\
\hline
\end{tabular}

Data are presented as median with interquartile range. Groups were compared using Kruskal-Wallis $\mathrm{H}$ test. Significance values have been adjusted by the Bonferroni correction for multiple comparisons. * Bonferroni adjusted $p=0.049$ vs. Late GDM, † Bonferroni adjusted $p=0.005$ vs. late GDM. A full list of all NBS analytes and their values is given in the Supplement Table 1. GDM=Gestational diabetes mellitus
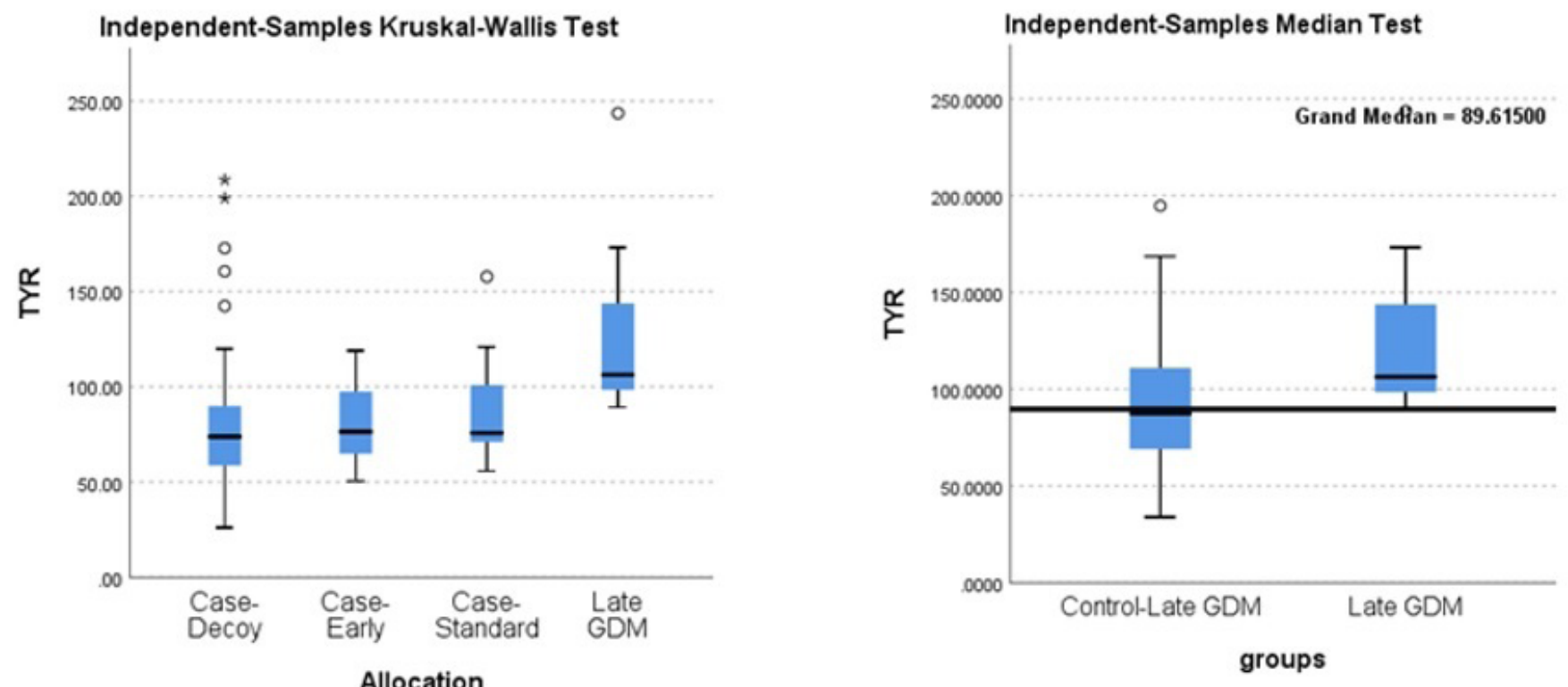

Figure 2. Comparison of tyrosine levels in the late GDM group versus early treatment, deferred treatment and decoy groups and between late GDM cases and controls. 
Table 3. Analytes of significance between late GDM and controls, early treatment and controls and decoy groups and controls.

\begin{tabular}{|c|c|c|c|}
\hline Analyte & Groups & Analyte level & $P$ value \\
\hline TYR $\mu \mathrm{mol} / \mathrm{l}$ & Late GDM vs. Late GDM controls & $\begin{array}{c}106.29(96.73-151.11) \text { vs. } \\
87.26(68.55-111.26)\end{array}$ & 0.01 \\
\hline IRT $\mu g \mathrm{gm} / \mathrm{I}$ & $\begin{array}{l}\text { Early treatment vs. Early treatment } \\
\text { controls }\end{array}$ & $\begin{array}{c}22.30(13.90-29.90) v s . \\
14(10.60-21.10)\end{array}$ & 0.02 \\
\hline $\mathrm{MET} \mu \mathrm{mol} / \mathrm{l}$ & Decoy vs. Decoy controls & $\begin{array}{l}30.33(24.74-35.51) \text { vs. } \\
27.07(22.64-32.27)\end{array}$ & 0.04 \\
\hline
\end{tabular}

Data are presented as median with interquartile range. Mann-Whitney $U$ was used to compare study groups against controls. A full list of all NBS analytes and their values is shown in the Supplementary material.

TYR=Tyrosine; IRT=Immunoreactive trypsinogen; MET=Methionine; GDM= Gestational diabetes mellitus; NBS=Newborn screening
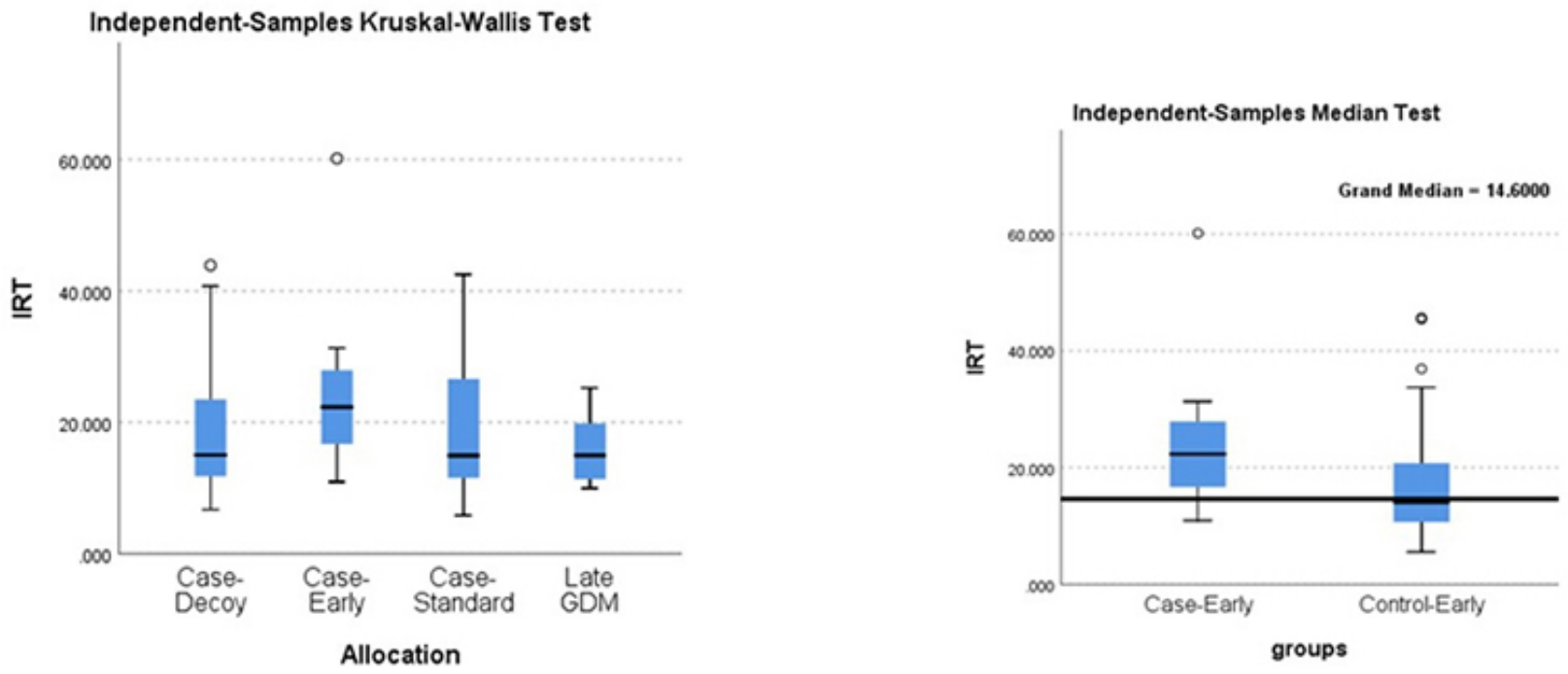

Figure 3. Comparison of Immunoreactive trypsinogen (IRT) between all groups, and between early treatment group and controls.
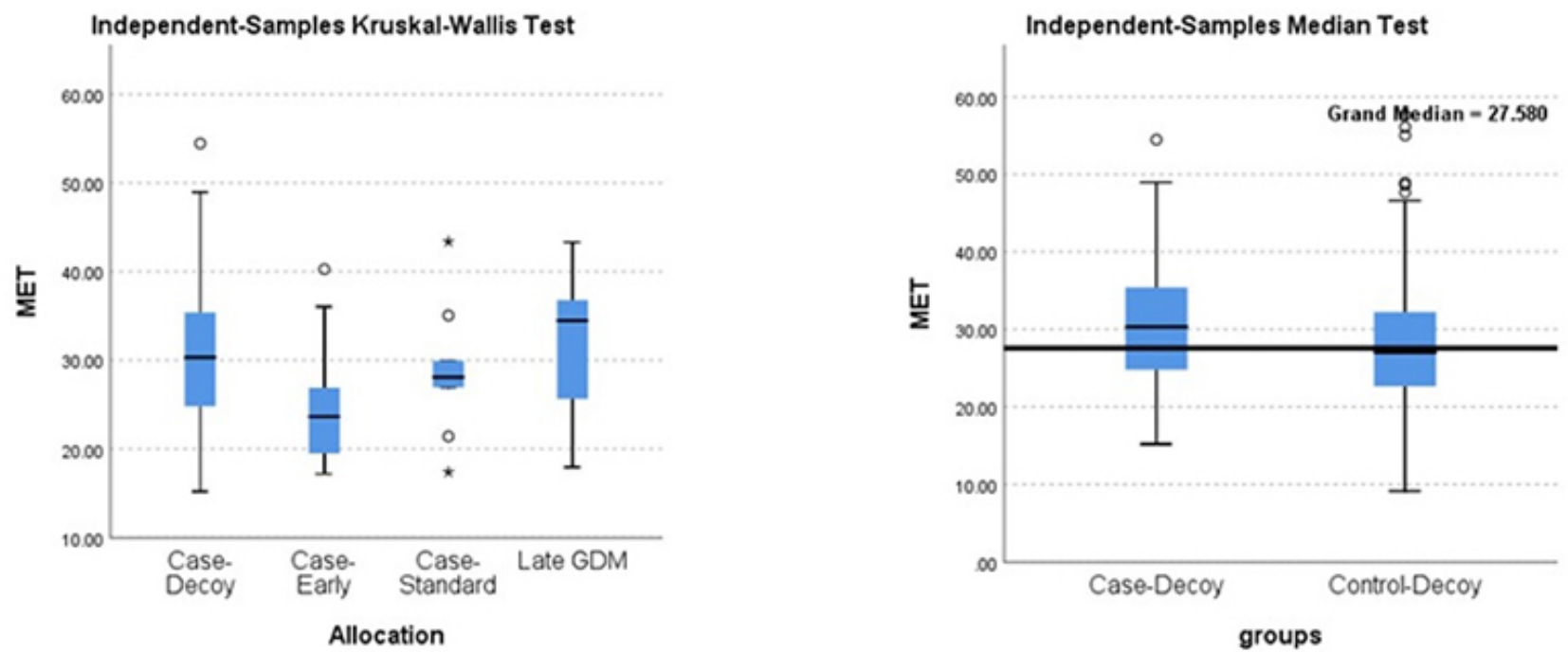

Figure 4. Comparison of Methionine between all groups, and decoy group and controls. 


\section{Discussion}

Our study specifically explores the association of diabetes in pregnancy and early treatment on infant metabolism through use of NBS collected 48-72 hours (majority at 48 hours) following delivery free from placental influence. Observing differences in how infants exposed to hyperglycemia in utero metabolize key nutritional substrates could increase understanding of the impact of GDM and its intergenerational consequences.

Tyrosine was the analyte that was consistently elevated when analyzed across four groups and when compared to controls. These levels were also higher than the median of the NSW NBS population for 2016 (whole blood $\mu \mathrm{mol} / 1 ;$ 79.44; 58.53-110.38). Tyrosine is a non-essential amino acid; higher levels have been associated with decreased insulin secretion and an increased risk for type 2 diabetes development in later life [23], and a negative association between tyrosine and cord blood c-peptide (a measure specific to fetal insulin secretion) has been reported [16]. This is not to imply causality, but that levels of tyrosine may be a surrogate marker of impaired glucose homeostasis. In addition to hormone synthesis, tyrosine is a key component of the insulin receptor substrate located in the cytosol. This protein contains 22 tyrosine residues that, once phosphorylated, provide a docking site for proteins involved in insulin-stimulated glucose uptake and expression of GLUT4 receptors. GDM exposure, despite good maternal glycemic control, may influence neonatal metabolism towards increased levels of an amino acid involved in glucose homeostasis.

IRT is a complex moiety of trypsin (from pancreatic acinar cells) largely bound to $\alpha-1$ antitrypsin. It is the only analyte tested for in NBS that gives an approximation of pancreatic (albeit exocrine) function. There is a physiologic rise in IRT levels in the first 24 hours of life, while transitioning from placental to enteral feeding [24]. IRT rises to actionable levels in perinatal stress, intercurrent illness, asymptomatic CF carrier infants [25] and SGA [26]. IRT levels were higher in infants in the early treatment group as compared to respective controls and higher than the NSW NBS population median (whole blood $\mu \mathrm{gm} / \mathrm{l}$; 17.1; 11.9-22.2), presumably due to the tendency of the group towards SGA. These findings were not seen in the deferred treatment, late treatment or decoy groups when compared with controls and it is possible that early treatment of GDM $(<20$ weeks) influences fetal programming of functions such as lipid synthesis. Brown fat development in the fetus begins around this time and supports our hypothesis that infants in the early treatment group tended towards being SGA.

Methionine is an essential amino acid involved in multiple metabolic processes in the body and associations with cardiometabolic disease have been described [27]. The decoy group, chosen for the presence of risk factors for GDM had higher MET levels. There is some evidence (animal and smallscale human trials) that MET restriction may be beneficial through increased energy expenditure and insulin sensitivity $[28,29]$, and if this association continues to be seen in large-scale human studies, it may be that treating hyperglycemia in future would entail some form of MET restriction. This would have to be very carefully considered in pregnancy, particularly with the metabolic demands of the maturing fetus.

Neonatal metabolism is shaped by many in-utero factors and the timing of neonatal exposure to treatment may be crucial in programming metabolic processes ex-utero. GDM per se may not be a single disorder and derangements observed in NBS results may reflect the timing of hyperglycemic exposure, treatment, and/or GDM heterogeneity [30-32].

Our study has several limitations: small sample size, and data were drawn from pilot study participants. The analytes measured were from one point in time and may not adequately represent the complexity of metabolic networks. Furthermore, ethnicity of the infant from whom the NBS sample is drawn is not recorded in NSW due to privacy concerns. This may be a source of bias, as ethnic-specific analyte trends have been observed in other work using cord blood $[16,17]$. We have not fully adjusted for multiple observations besides using the Bonferonni test which may be too conservative, but our initial results provide the basis for hypothesis generation. A larger sample size will clarify any statistical uncertainty and we plan to undertake analysis on the main TOBOGM trial cohort (predicted to be approx. 800) in NSW [19]. We were unable to perform matching of maternal characteristics of the age-matched controls chosen from the NSW population which may have potential confounders, but the ratio of cases to controls used, and the consistency of the findings provides a degree of reassurance that the chosen controls do reflect a normal population. The observational nature of the study may not have accounted for unmeasured confounding. In addition, maternal glucose control during pregnancy was not easily available: there are no reliable methods for assessing control other than regular fingerprick testing and recording, which may be a confounder. However, treatment was escalated when women had three readings off-target in a week, which provides a degree of reassurance that glucose levels were within treatment range.

The primary purpose of NBS is, and should remain, as screening for conditions that present and require treatment early in life with serious consequences and reasonable frequency. However, the nature of newborn screening allows a valuable opportunity to evaluate effects of in-utero exposure on neonatal metabolism free from placental influence and a constant nutritional supply.

\section{Authors' Note}

These results form part of the data from the treatment of booking gestational diabetes mellitus (TOBOGM) pilot randomised controlled trial Australia New Zealand Clinical Trials Registry ACTRN12615000974505. Registered 17th May 2015; URL: https://www.anzctr.org.au/trial/registration/trialreview. aspx?id=369100\&isreview=true

\section{Funding}

JI is supported by a postgraduate research scholarship from Western Sydney University. 


\section{Authors' Contributions}

JE and VW conceived the newborn screening part of the pilot project. JE and JI performed statistical analysis. JE interpreted the data and drafted the manuscript. DS conceived the TOBOGM pilot project and together with VW, supervised JE, interpreted the data, reviewed and edited the draft and provided critical input to the manuscript. All authors read and approved the final manuscript. DS is the guarantor of this work and, as such, had full access to all the data in the study and takes responsibility for the integrity of the data and the accuracy of the data analysis.

\section{Declaration of Conflicting Interests}

The authors declared no potential conflicts of interest with respect to the research, authorship, and/or publication of this article.

\section{Supplementary Material}

The following online material is available for this article:

Table S1 - Comparison of newborn screening analytes according to TOBOGM randomization.

Table S2 - Early treatment cases vs. controls.

Table S3 - Comparison of deferred treatment cases with NSW controls.

Table S4 - Comparison of late GDM treatment cases with NSW controls.

Table S5 - Comparison of decoys versus NSW controls.

\section{References}

1. Wilson K, Hawken S, Potter BK, et al. Accurate prediction of gestational age using newborn screening analyte data. Am JObstet Gynecol. 2016;214(4):513.e1-513.e9. doi:10.1016/j. ajog.2015.10.017

2. Sylvester KG, Kastenberg ZJ, Moss RL, et al. Acylcarnitine profiles reflect metabolic vulnerability for necrotizing enterocolitis in newborns born premature. J Pediatr. 2017;181:80-85.e1. doi:10.1016/j.jpeds.2016.10.019

3. Ryckman KK, Berberich SL, Dagle JM. Predicting gestational age using neonatal metabolic markers. Am J Obstet Gynecol. 2016;214(4):515.e1-515.e13. doi:10.1016/j. ajog.2015.11.028

4. Jelliffe-Pawlowski LL, Norton ME, Baer RJ, et al. Gestational dating by metabolic profile at birth: a California cohort study. Am J Obstet Gynecol. 2016;214(4):511.e1-511.e13. doi:10.1016/j.ajog.2015.11.029

5. Rusconi B, Warner BB. The hidden treasure of neonatal screening: Identifying new risk factors and possible mechanisms of necrotizing enterocolitis through Big Data. J Pediatr. 2017;181:9-11. doi:10.1016/j.jpeds.2016.11.028
6. Sanchez-Pintos P, de Castro MJ, Roca I, et al. Similarities between acylcarnitine profiles in large for gestational age newborns and obesity. Sci Rep. 2017;7(1):16267. doi:10.1038/s41598-017-15809-4

7. Wright EL, Baker PR. Neonatal macrosomia is an interfering factor for analytes on the Colorado state newborn screen. JClin Endocrinol Metab. 2020;105(3):dgz183. doi:10.1210/ clinem/dgz183

8. Kyvsgaard J, Overgaard AJ, Thorsen SU, et al. High neonatal blood iron content is associated with the risk of childhood type 1 diabetes mellitus. Nutrients. 2017;9(11):1221. doi:10.3390/nu9111221

9. McDonald TJ, Besser RE, Perry M, et al. Screening for neonatal diabetes at day 5 of life using dried blood spot glucose measurement. Diabetologia. 2017;60(11):21682173. doi:10.1007/s00125-017-4383-3

10. Cadario F, Savastio S, Pagliardini V, et al. Vitamin D levels at birth and risk of type 1 diabetes in childhood: a case-control study. Acta Diabetol. 2015;52(6):1077-1081. doi:10.1007/ s00592-015-0772-6

11. Jacobsen R, Thorsen SU, Cohen AS, et al. Neonatal vitamin $D$ status is not associated with later risk of type 1 diabetes: results from two large Danish population-based studies. Diabetologia. 2016;59(9):1871-1881. doi:10.1007/s00125016-4002-8

12. Kyvsgaard JN, Overgaard AJ, Jacobsen LD, et al. Low perinatal zinc status is not associated with the risk of type 1 diabetes in children. Pediatr Diabetes. 2016;18(7):637-642. doi:10.1111/pedi.12476

13. la Marca G, Malvagia S, Toni S, et al. Children who develop type 1 diabetes early in life show low levels of carnitine and amino acids at birth: Does this finding shed light on the etiopathogenesis of the disease? Nutr Diabetes. 2013;3:e94. doi:10.1038/nutd.2013.33

14. Simaite D, Kofent J, Gong M, et al. Recessive mutations in PCBD1 cause a new type of early-onset diabetes. Diabetes. 2014;63(10):3557-3564. doi:10.2337/db13-1784

15. Lowe WL Jr, Bain JR, Nodzenski M, et al. Maternal BMI and glycemia impact the fetal metabolome. Diabetes Care. 2017;40(7):902-910. doi:10.2337/dc16-245

16. Kadakia R, Scholtens DM, Rouleau GW, et al. Cord Blood Metabolites Associated with Newborn Adiposity and Hyperinsulinemia. J Pediatr. 2018;203:144-149.e1. doi: 10.1016/j.jpeds.2018.07.056

17. Nankervis A, McIntyre HD, Moses R, et al. Australian Diabetes in Pregnancy Society (ADIPS) Consensus Guidelines for the Testing and Diagnosis of Gestational Diabetes Mellitus in Australia. 2013. http://www.adips.org/ downloads/adipsconsensusguidelinesgdm-03. 05.13versionacceptedfinal.pdf 
18. Immanuel J, Simmons D. Screening and treatment for early-onset gestational Diabetes Mellitus: A systematic review and meta-analysis. Curr Diab Rep. 2017;17(11):115. doi:10.1007/s11892-017-0943-7

19. Simmons D, Nema J, Parton C, et al. The treatment of booking gestational diabetes mellitus (TOBOGM) pilot randomised controlled trial. BMC Pregnancy Childbirth. 2018;18(1):151. doi:10.1186/s12884-018-1809-y

20. Human Genetics Society of Australasia. Policy: Recommendations for Screening for Specific Disorders. 2017. https://www.hgsa.org.au/documents/item/8694. Accessed April 24, 2020.

21. NSW Health, Office of Kids and Families. Newborn bloodspot screening. Section 8.2.2., p. 9. May 18, 2016. https://www1.health.nsw.gov.au/pds/activepdsdocuments/ pd2016_015.pdf. Accessed April 24, 2020.

22. Wilcken B, Wiley V. Newborn screening. Pathology. 2008;40(2):104-115. doi:10.1080/00313020701813743

23. Vangipurapu J, Stancáková A, Smith U, et al. Nine amino acids are associated with decreased insulin secretion and elevated glucose levels in a 7.4-Year follow-up study of 5,181 Finnish men. Diabetes. 2019;68(6):1353-1358. doi:10.2337/ db18-1076

24. Heeley AF, Bangert SK. The neonatal detection of cystic fibrosis by measurement of immunoreactive trypsin in blood. Ann Clin Biochem. 1992;29(Pt 4):361-376. doi:10.1177/000456329202900401

25. Massie J, Curnow L, Tzanakos N, et al. Markedly elevated neonatal immunoreactive trypsinogen levels in the absence of cystic fibrosis gene mutations is not an indication for further testing. Arch Dis Child. 2006;91(3):222-225. doi:10.1136/adc.2005.081349

26. Wilcken B. Newborn screening for cystic fibrosis: Its evolution and a review of the current situation. Screening. 1993;2(1):43-62. doi:10.1016/0925-6164(93)90017-d

27. Olsen T, Øvrebø B, Haj-Yasein N, et al. Effects of dietary methionine and cysteine restriction on plasma biomarkers, serum fibroblast growth factor 21 , and adipose tissue gene expression in women with overweight or obesity: a doubleblind randomized controlled pilot study. J Transl Med. 2020;18:122. doi:10.1186/s12967-020-02288-X

28. Castaño-Martinez T, Schumacher F, Schumacher S, et al. Methionine restriction prevents onset of type 2 diabetes in NZO mice. FASEB J. 2019;33(6):7092-7102. doi:10.1096/ fj.201900150R

29. Orgeron ML, Stone KP, Wanders D, et al. The impact of dietary methionine restriction on biomarkers of metabolic health. Prog Mol Biol Transl Sci. 2014;121:351-376. doi:10.1016/B978-0-12-800101-1.00011-9

30. Powe CE, Allard C, Battista MC, et al. Heterogeneous contribution of insulin sensitivity and secretion defects to gestational diabetes mellitus. Diabetes Care. 2016;39(6):1052-1055. doi:10.2337/dc15-2672

31. Immanuel J, Simmons D, Harreiter J, et al. (2020), Metabolic phenotypes of early gestational diabetes mellitus and their association with adverse pregnancy outcomes. Diabet Med. 2021;38(2):e14413. doi:10.1111/dme.14413

32. Egan AM, Simmons D. Lessons learned from lifestyle prevention trials in gestational diabetes mellitus. Diabet Med. 2019;36(2):142-150. doi:10.1111/dme.13772 